\title{
足球动态传球网络的可视化
}

\author{
谢潇1)，梁鸿业 ${ }^{2}$ ，巫英才 ${ }^{2}$ * \\ 1) (浙江大学教育学院体育学系 杭州 310058) \\ 2) (浙江大学 CAD\&CG 国家重点实验室 杭州 310058) \\ (ycwu@zju.edu.cn)
}

\begin{abstract}
摘 要：足球传球网络分析对提高球队的进攻效率起着重要作用. 然而, 大多数现有工作都将传球网络视为静态网 络, 忽略了比赛过程中传球的时空演变. 该工作通过与领域专家的深人访谈总结了动态传球网络分析的重要任务, 并提出了一个交互式可视分析系统支持对传球网络的时空动态性进行全面分析. 该系统应用了基于图标的可视化方 法和投影技术，以呈现不同时间下的传球网络拓扑结构和传球空间上下文信息. 该系统可以辅助用户发现传球网络 结构和传球所在的空间位置在时间上的变化模式以及两者之间的关联，并根据传球网络动态性对比赛过程进行更深 人的解读, 发现有价值的传球网络变化策略. 通过案例分析和专家访谈, 验证了该系统在分析传球网络动态性的可 用性和有效性.
\end{abstract}

关键词：体育可视化; 足球传球网络; 动态网络; 可视分析

中图法分类号: TP391.41 DOI: 10.3724/SP.J.1089.2021.19267

\section{Toward Better Understanding of Dynamic Soccer Passing Networks}

\author{
Xie Xiao ${ }^{1)}$, Liang Hongye ${ }^{2)}$, and Wu Yingcai ${ }^{2)^{*}}$ \\ 1) (Department of Sports Science, College of Education, Zhejiang University, Hangzhou 310058) \\ 2) (State Key Laboratory of CAD\&CG, Zhejiang University, Hangzhou 310058)
}

\begin{abstract}
Recent studies have confirmed that analysis of passing networks of soccer can provide insights for understanding teams' attacking strategies. However, most of the existing works regarded passing networks as static networks, failing to investigate the change of strategies and time-varying passing patterns. To address the problem, important tasks from interviews with domain experts are identified and an interactive visual analytics system is developed to support comprehensive analysis of the dynamics of passing networks. A glyph-based design accompanied with projection techniques is proposed to visualize the dynamic change in network topologies, the associated spatial context, and the network characteristics. With the system, users can detect the changing pattern of passes and analyze the relationship between passes and spatial regions. For evaluation, expert interviews are conducted to demonstrate the usability of the system in analyzing the dynamics of passing networks.
\end{abstract}

Key words: sports visualizations; soccer passing networks; temporal networks; visual analytics

足球是世界上最受欢迎的运动之一，有着广 泛的影响力和重要的商业价值. 因此，大量分析师
尝试通过分析足球数据帮助球队提高表现. 传球 分析是其中一类重要的分析方向，其典型方法是

收稿日期：2021-07-08; 修回日期：2021-09-13．基金项目：国家自然科学基金面上项目(62072400); 浙江省自然科学基金杰出青 年基金(LR18F020001). 谢潚(1994一), 男, 博士, 特聘研究员, 主要研究方向为体育数据可视化、人机交互、可视分析; 梁鸿业 (1996一), 男, 硕士, 主要研究方向为体育数据可视化; 巫英才(1983-), 男, 博士, 教授, 论文通讯作者, 主要研究方向为可视分析、 信息可视化、人机交互. 
将球员作为网络节点, 将球员传球作为边, 构造出 一个传球网络进行分析. 这类分析常见于比赛的 赛后报告中(如 Opta 和 STATS). 研究者通过应用 网络分析技术发现传球网络的特征，如根据出人 度发现关键球员，根据边发现常见的传球模式，根 据路径判断球队传球的流畅度等.

完成此类分析需要聚合比赛中的所有传球记 录构造出一个静态的传球网络. 但是, 这一聚合方 法忽略了传球的动态性, 阻碍了对战术变化的深 人研究. 传球是随时间变化的行为, 取决于多种因 素, 如球员的表现和替换. 例如, 上半场和下半场 的传球战术可能因为换人而出现巨大变化，比分 领先时与比分落后时的传球行为也有着重大的区 别. 聚合这一数据处理步骤导致传统传球网络分 析技术难以揭示此类战术调整. 因此, 专家们迫切 地希望对传球的动态性进行深人分析.

近年来, 传球动态性的研究逐渐受到重视. Yamamoto 等 ${ }^{[1]}$ 以 $5 \mathrm{~min}$ 为间隔对比赛进行划分, 并 以折线图的形式可视化每段的传球网络中包含的 三角形结构的数量, 进而分析传球动态性. 但是, 该方法依赖分割的时间间隔，缺乏灵活性，难以被 广泛应用. PassVizor ${ }^{[2]}$ 从序列的角度对传球动态性 进行分析，发现了球员传球组合随时间的变化模 式. 但是, 球员真实的传球顺序难以体现, 阻碍了 对具体传球配合的分析. 如何从网络的角度分析 足球传球的动态性仍有待解决.

可视化由于其直观性，在体育分析中已经得 到了广泛的应用. 因此, 本文将应用可视化技术对 动态的传球网络进行深人分析. 动态网络的可视 化一直是可视化研究的热点. 例如, 文献[3]通过 时间窗口的方法获得每个时间片段上的网络结构, 并以邻接矩阵的形式对网络进行向量化. 基于投 影算法, 文献[3]将多个子网络映射到一维或二维 空间, 通过连接相邻时间的子网络刻画出时间轴, 用户通过子网络映射位置的变化可以发现网络结 构随时间的演变模式. 但是, 传球网络除了在拓扑 结构上存在变化外, 节点(即球员)的空间位置也会 随时间发生改变. 因此, 已有的动态网络可视化方 法难以被直接应用于动态传球网络的展示.

本文开发了一个交互式的可视分析系统，以 支持网络角度的动态传球分析. 在开发过程中, 通 过与领域专家的 6 个月的合作总结了领域需求, 采 用了图标可视化与投影技术结合的方法实现了足 球动态传球网络的可视呈现, 并根据需求设计了 包含多个联动视图的可视分析系统支持分析. 本
文的主要贡献包括: (1) 给出动态传球网络分析的 领域需求; (2) 结合图标可视化和投影技术解决动 态传球网络的呈现问题; (3) 实现交互式的动态传 球网络可视分析系统.

\section{1 相关工作}

\section{1 足球传球网络分析}

近年来, 通过应用传统的网络分析技术, 分析 人员提出了一系列针对足球传球网络的分析工作. 大量工作 ${ }^{[4-6]}$ 通过计算常见的网络统计指标来发现 传球数据中潜藏的模式. 例如, Peña 等 ${ }^{[6]}$ 根据西班 牙队传球网络计算了球员的度中心性, 结果表明, 西班牙队的传球更为均衡, 所有球员都有效地参 与到传球中. Clemente 等 ${ }^{[5]}$ 采用了统计检验的方法 验证了球员的位置, 如前锋、中场等和其对应的网 络指标之间的关系, 研究发现, 中场球员为球队进 攻做出了最突出的贡献.

一批研究者采用了基于模式的方法 ${ }^{[7-9]}$ 分析球 员之间的传球模式. Gyarmati 等 ${ }^{[8]}$ 定义了 4 类传球 模式, 并根据不同球队的传球模式分布进行聚类, 结果表明, 巴塞罗那俱乐部与其他球队的传球模 式有着显著不同，局部球员之间存在着频繁的互 相传球的现象. Bekkers 等 ${ }^{[7]}$ 使用传球模式分布对 球队和球员的比赛风格进行表征, 分析了球队成 员的变化对球队传球风格的影响.

然而, 以上工作都是对静态的传球网络进行 分析, 无法发现传球随时间的变化. 认识到这一缺 陷后, 部分工作 ${ }^{[1,10-13]}$ 提出了动态的分析方法. Cotta 等 ${ }^{[11]}$ 和 Yamamoto 等 ${ }^{[1]}$ 基于时间窗口对网络 进行划分, 通过网络的统计指标变化发现传球的 动态性. 但是, 网络指标往往只能反映网络的部分 特征, 难以支持全局的探索分析. 同时, 此类方法 还无法支持传球网络拓扑结构和传球时空间上下 文的联动分析, 阻碍了传球动态性的发现和解读. 因此, 本文开发了一个交互式的可视分析系统以 解决此问题.

\section{2 动态网络可视化}

动态网络可视化在可视化领域受到了广泛的 关注. Beck 等 ${ }^{[14]}$ 通过调研分析将动态网络可视化 分为基于动画的方法和基于时间线的方法.

基于动画的方法 ${ }^{[15-16]}$ 利用动画的直观性帮助 用户更好地理解网络结构的变化. Forbes 等 ${ }^{[17]}$ 通过 动画的形式展示蛋白质结构中节点和边的出现和 消失, 促进对蛋白质结构演变的理解. 尽管动画有 
着直观易懂的优点，但用户在长时间观看动态网 络的动画后会产生严重的认知负担 ${ }^{[18]}$.

基于时间线的方法将不同时间段的网络放置 在时间线上展示动态性. 其中, 一项典型工作 (如 egoSlider ${ }^{[19]}$ )通过设计的图标展示每个时间段上的 自我中心网络，并利用时间线说明网络的变化. Zhao 等 ${ }^{[20]}$ 进一步发明了基于地铁线路图隐喻的动 态图可视化说明具体的拓扑变化. 但是, 自我中心 网络的结构与传球网络相比较为简单, 专家探索 的不仅限于邻近的关系, 还涉及多个球员的传球 路径关系. 因此，以上方法无法被直接应用. 部分 研究人员专注于整体网络动态性的可视呈现 ${ }^{[21-22]}$. Greilich 等 ${ }^{[23]}$ 将每个时间段的网络在垂直方向上进 行布局, 并将不同时间段上的节点相连以显示变 化. 然而, 当使用该方法展示时间跨度较长的动态 网络时, 用户难以长时间跟踪网络变化. 为此, 文 献[3]将网络的每个时间段的网络向量化并映射到 二维空间中的一个点, 通过时间相邻点的位置变 化表示网络的动态性. 但是，每次传球的空间上下 文信息, 如传球的位置和接球的位置, 对于识别和 理解球员的传球策略在传球网络的分析中是至关 重要的. 因此，本文结合了投影方法和基于图标的 技术，可视化传球网络的拓扑演变以及相关空间 上下文信息

\section{2 分析传球动态性的可视分析方法}

\section{1 数据背景}

本文所使用的数据为 U15 比赛数据, 分别为 阿根廷对巴西和阿根廷对秘鲁. 分析师使用半自 动采集系统 ${ }^{[24]}$ 从比赛视频中采集了比赛数据.

数据记录了每时刻场上球员和足球的空间位 置和比赛事件以及计算得到的传球次数、防守面 积、跑动距离、冲刺速度、冲刺距离等.

\section{2 需求分析}

R1. 传球网络的拓扑结构随时间的演变. 球 队的传球风格可以通过传球网络的拓扑结构来表 示. 跟踪拓扑结构的演变可以帮助专家了解比赛 策略的动态改变和教练的决策过程.

R2. 传球网络在空间上的分布. 除了拓扑结 构外, 专家还渴望了解传球网络所覆盖的球场空 间区域，以了解主要的进攻策略和模式. 同时，专 家还需要知道球员何时在特定的空间区域(如中 场)进行传球配合.
R3. 传球拓扑结构和空间区域的联系. 这可 以帮助专家发现球队在不同空间区域是否有着不 同传球配合的倾向性，从而确定球队在每个区域 的传球偏好，为策略的制定提供建议.

R4. 传球网络特征随时间的演变规律. 揭示 传球网络的特征如传球次数和参与球员的数量可 以帮助专家了解传球网络的状态和影响, 而且, 可 以使专家更好地了解传球网络的比赛表现, 找到 有利于球队进攻的传球战术.

R5. 球员在传球网络中的表现. 衡量球员在 传球网络中的表现可以帮助专家发现核心球员和 表现不佳的球员, 从而理解球员的优势、劣势以及 与团队策略的适应性. 这可以进一步促进如阵容 选择和球员替换等任务.

\section{3 系统设计}

根据需求分析结果, 本文开发了一个如图 1 所 示的交互式的可视分析系统，其包含了 3 个视图: 演化视图(A 部分), 使用投影的技术可视化比赛中 随时间变化的传球网络; 网络视图(B 部分), 使用 聚合的点线图展示整个传球网络和球员个人的临 场表现; 球场视图(C 部分), 利用了二维球场和点 线图展示指定传球子网在球场上的具体位置和上 下文信息.

考虑一致性，系统将比赛球队的进攻方向统 一设定为从球场左侧至球场右侧, 球员和球的空 间位置也进行了相应的转换处理.

\subsection{1 演化视图}

该视图采用了投影方法将每个回合的传球网 络向量化后投影到一维呈现传球网络拓扑结构的 动态性(R1). 网络向量化的方法可参照文献[3]的 工作. 如图 1 中 A3 部分所示, 圆形的图标代表了 一个真实的传球网络. 图标在水平方向上的位置 编码了该传球网络对应的比赛时间, 左边是比赛 起点, 右边是比赛终点. 图标在垂直方向上的位置 编码了传球网络的拓扑结构的相似性, 2 个图标在 垂直方向上的位置相差越远, 说明两者的网络结 构越不相似; 反之, 则越相似. 与传统的投影方法 相同 ${ }^{[3]}$, 图标在垂直方向上的绝对位置没有实际意 义. 比赛的上下半场的传球网络通过间隔进行区 分(图 1 中 A2 部分). 每个传球网络的下方都放置 了代表比赛事件的图标(图 1 中 A1 部分), 帮助专 家快速发现传球的结果. 专家可以将鼠标悬浮在 事件图标上, 具有相同事件的传球网络会被高亮 显示，以支持更深人的对比分析. 


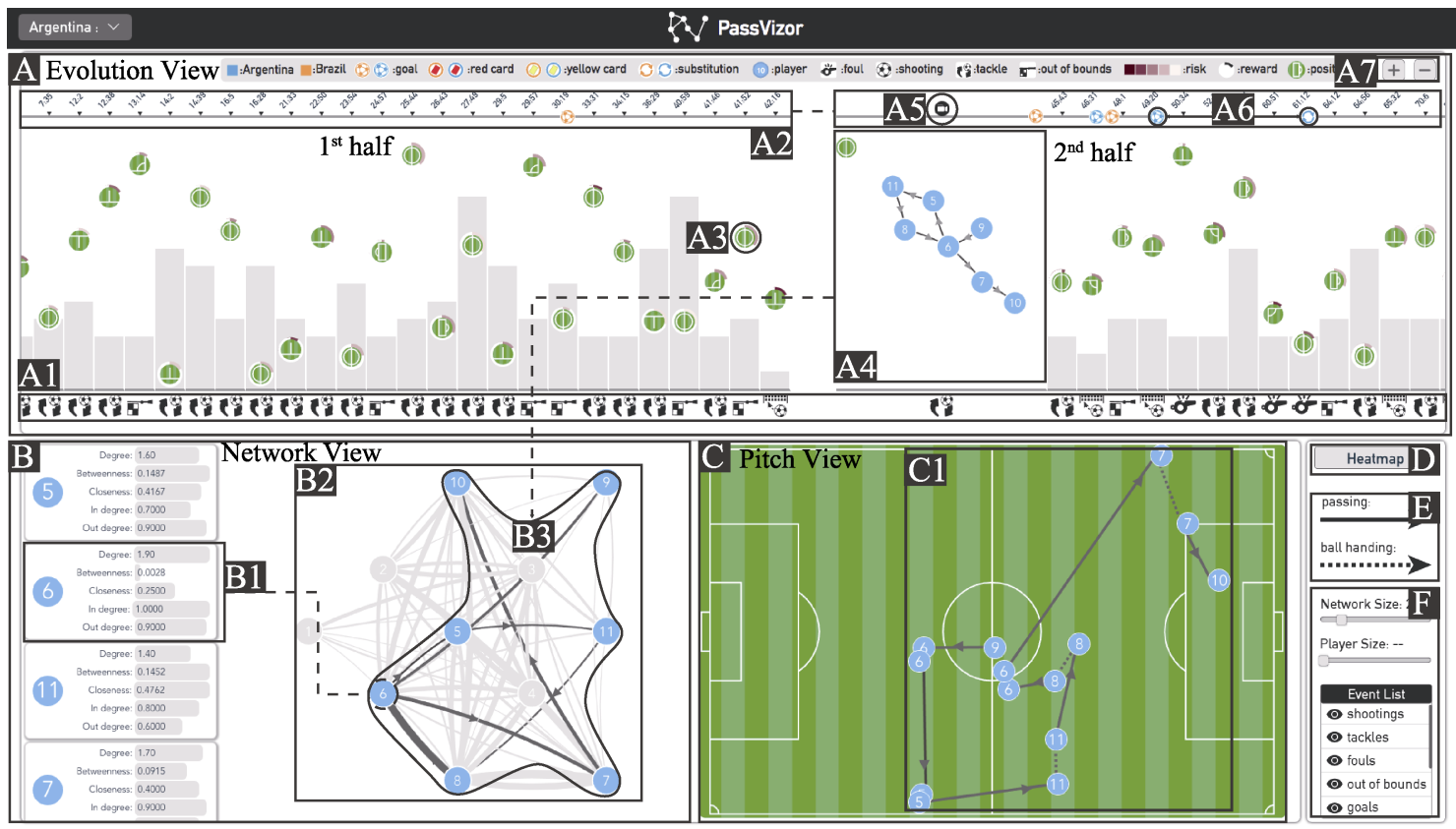

图 1 足球动态传球可视分析系统界面

为了表示传球所在的空间位置, 本文采用了 PassVizor ${ }^{[2]}$ 中的球场空间图标设计, 将足球比赛场 地分为 9 块, 使用区域中最突出的视觉特征表示该 区域. 例如，使用球场的中圈图案代表球场中间的 这块区域(图 2 中 $\mathrm{A}$ 部分). 该图案被放置在传球图 标的中心(图 1 中 A3 部分)以表示某一传球网络中 球员传球最频繁的空间区域 (R2, R3). 通过观察 空间图标在时间上的变化, 专家可以发现球队进 攻方向在空间上的转移，以及这种空间转移与网 络的拓扑结构之间是否存在联系. 传球图标所在 的垂直空间内的柱状图编码了对应传球网络中的 总传球次数, 柱状图高度越高, 表示传球次数越 多. 这一设计可以帮助专家发现传球流畅的回合 并相应地进行重点分析. 传球图标外的环形图编 码了传球网络的收益值和风险值(R4). 收益值表 示传球网络创造出射门机会的概率，使用环形图 的弧长表示, 弧度越长, 概率越大. 风险值表示传 球网络造成丢球的概率，使用环形图的颜色表示， 颜色越深, 风险越高, 具体的编码规则可见图 2 中 B 部分和 C 部分. 本文使用了 Power 等 ${ }^{[25]}$ 的方法计 算每次传球的收益和风险，并使用每个传球网络 中的所有传球行为中的收益最大值和风险最大值 代表整个传球网络.

演化视图支持如下交互操作. (1) 过滤传球网 络. 用户可以根据不同的属性，如空间位置和参与 传球的球员等, 对传球网络进行过滤, 以进行更具 目标性的分析(图 1 中 $\mathrm{F}$ 部分). 篮选选项包括传球
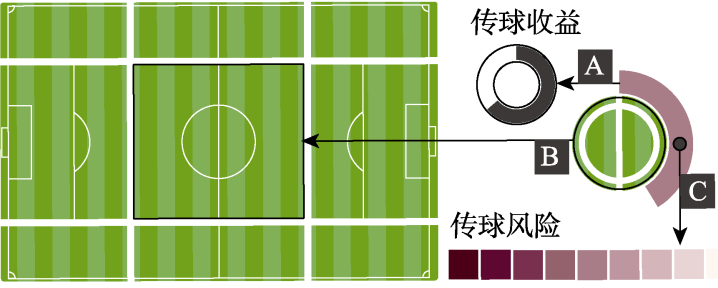

图 2 传球网络图标设计

网络包含的球员数量、传球网络所在的空间位置和 传球序列的结束事件. (2) 显示拓扑结构. 用户可 以点击代表传球网络的图标，对应的具体拓扑结 构将会以点线图的形式展示(图 1 中 A4 部分). 当 用户将鼠标悬停在传球子网图标上时, 所有具有 相同空间区域的传球子网图标将会高亮显示, 以 帮助用户确定整场比赛的传球网络的空间分布. (3) 查看原始视频. 用户可以点击对应的视频按钮查看 某一传球网络对应的原始视频(图 1 中 A5 部分).

\subsection{2 网络视图}

网络视图展示的是整场比赛中传球网络的整 体拓扑结构(R4, R5). 如图 1 中 B2 部分所示, 球员 按照阵型进行排列, 球员之间边的粗细表示了传 球次数. 用户可以点击某一球员, 相应的网络指标 会在左侧显示(图 1 中 B1 部分). 当用户在演化视 图中点击了某一传球网络的图标时，该子网络会 在网络视图中高亮显示(图 1 中 B3 部分). 这可以 帮助专家发现该子网是否是球队常规的配合模式.

\subsection{3 球场视图}

球场视图以二维的形式展示了某一传球子网 
在球场上具体的空间分布(图 1 中 C1 部分). 用户 可以在演化视图中点击传球网络图标选中该子网. 实线表示了球员间的传球行为，而虚线表示了球 员的带球行为. 用户可以进一步点击热力图的按 钮(图 1 中 D 部分), 查看整场比赛下球员在传球时 频繁活动的范围, 并结合某一具体的传球子网进 行分析.

\section{3 案例分析}

本文邀请了 2 位专家使用该系统对比赛数据 (阿根廷对阵巴西的 U15 足球锦标赛)中的传球进 行深人分析，通过案例分析和专家反馈对该系统 进行评估. 2 位专家包括 1 名有超过 10 年经历的高 级体育分析师和 1 名获得亚足联 $\mathrm{A}$ 级教练证书的 高级教练. 评估的流程如下: (1) 为专家提供系统使 用的详细介绍; (2) 专家使用该系统进行案例分析; (3) 收集专家的反馈和建议. 案例和反馈总结如下.

\section{1 传球网络的概览分析}

专家们首先在演化视图中对呈现的传球网络 进行篮选, 以了解球队如何通过传球组织进攻. 因 此, 过滤了没有产生射门事件且传球次数较少的 传球网络(图 3 中 $\mathrm{F}$ 部分). 随后, 在演化视图中发 现了上下半场的传球网络特征之间存在着差异.
其中, 在下半场(图 3 中 A 部分和 B 部分), 无论是 传球网络的回合数量(传球图标的数目), 还是传球 网络中传球的次数(柱状图的高度), 都出现了明显 下降. 在结合比赛事件后, 专家认为, 阿根廷在上 半场的战术是主动发起进攻, 尝试把握比赛的主 动权, 因此出现了较多的进攻回合和传球次数. 但 是，这一策略没有取到很好的效果. 结合事件列表 (图 3 中 A1 部分)可以看到, 上半场多个进攻回合 中只有少数成功地造成了射门. 专家们判断, 阿根 廷在中场发现了这一现象并认为原进攻策略无法 有效地突破对方的防守, 因此决定在下半场采取 偏保守的策略，将球权更多地交给对手，尝试通过 抓住对手进攻上的失误获得进球机会. 为了验证 这一猜想, 专家对下半场的传球进行了分析. 如图 3 中 B2 部分和 B4 部分所示, 在查看下半场的传球 网络时, 专家们发现传球图标的弧圈比上半场有 着更长的弧度和更深的颜色, 说明大部分传球的 收益和风险都比较低. 由此, 专家们确认了阿根廷 在下半场倾向于更保险的传球方式, 策略转变为 通过防守反击抓住对手的失误完成关键传球进而 创造得分的机会(R4). 从比赛事件上专家们发现, 阿根廷在下半场攻人 3 个球并最终赢得了比赛，说 明了这一传球策略的调整有效地帮助阿根廷获得 了比赛的胜利.

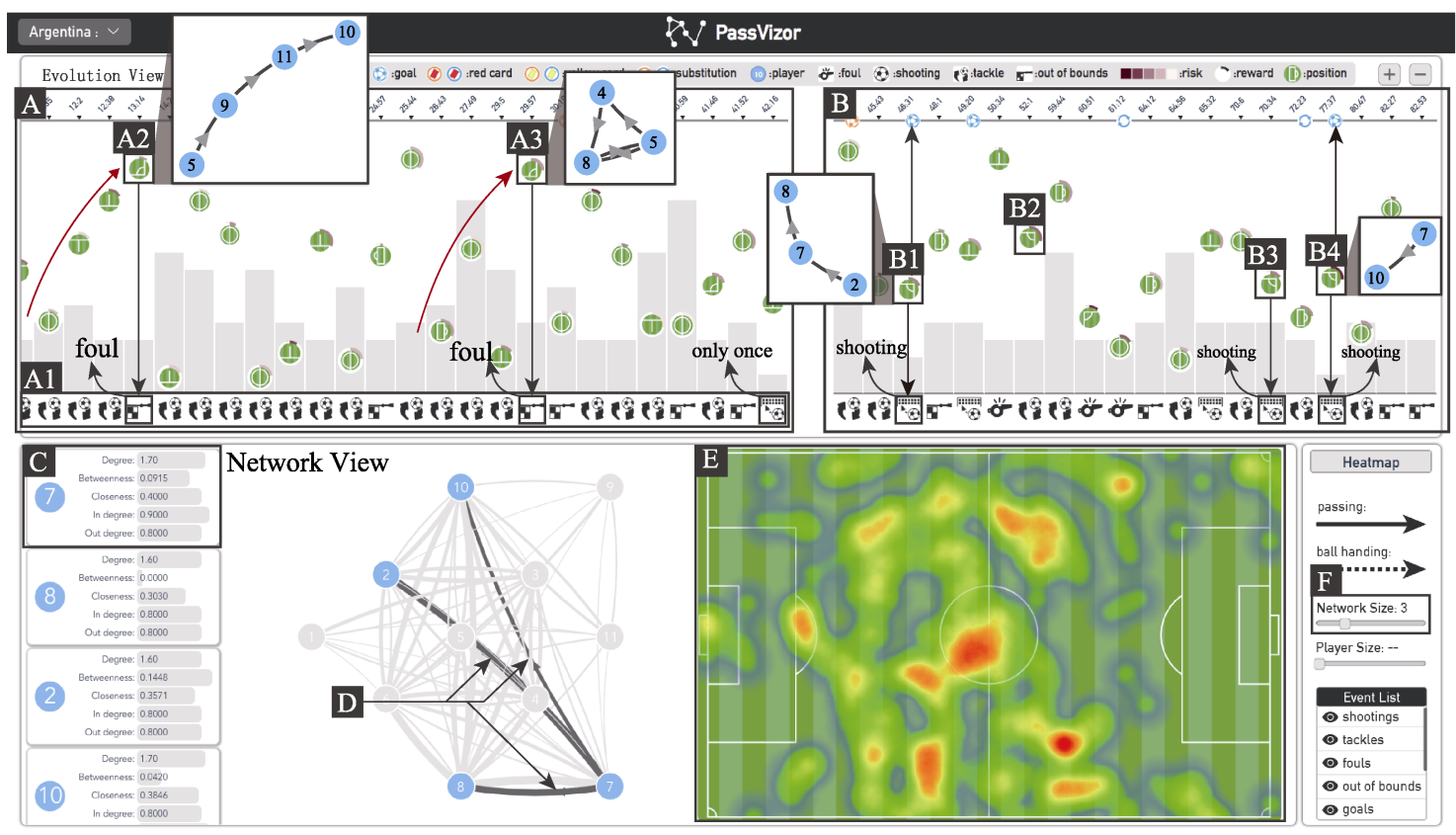

图 3 足球动态传球案例分析

\section{2 传球网络的动态分析}

上述分析从宏观的角度发现了阿根廷上下半 场的传球策略的改变. 为了更清楚地了解球队是
如何践行对应的传球策略的, 专家们专注于分析 上下半场传球网络图标的不同，以及随时间的演 变趋势. 如图 3 中 A2 部分的红色箭头所示, 发现 
传球网络图标在垂直方向上有连续的上升趋势. 这说明了传球网络结构以一定的方式进行演变 (R1). 同时，从传球网络图标中间代表空间区域的 图案可以观测到传球所在的空间区域逐渐向球场 的右上角区域移动. 在后续的图标中同样发现了 这一种传球区域向右上角转移的模式(图 3 中 A3 部分). 基于以上的现象, 专家们认为, 阿根廷在 这段时间内的主要进攻区域是球场右侧, 并通过 多次的球的转移达到这一目的(R2, R3). 专家们随 后分别查看了 2 个右上角区域的传球网络(图 3 中 A2 部分和 A3 部分), 经对比后发现两者有着类似 的传球拓扑结构(图 4 中 A 部分). 这也验证了先前 的猜想. 为了进一步确认这一传球进攻模式起到 的效果, 专家们查看了对应的事件列表, 发现 2 次 进攻均以被抢断结束(图 3 中 A1 部分). 因此, 专家 认为对方的防守策略可以有效地应对来自右侧的 进攻, 并通过原始视频进行了确认.

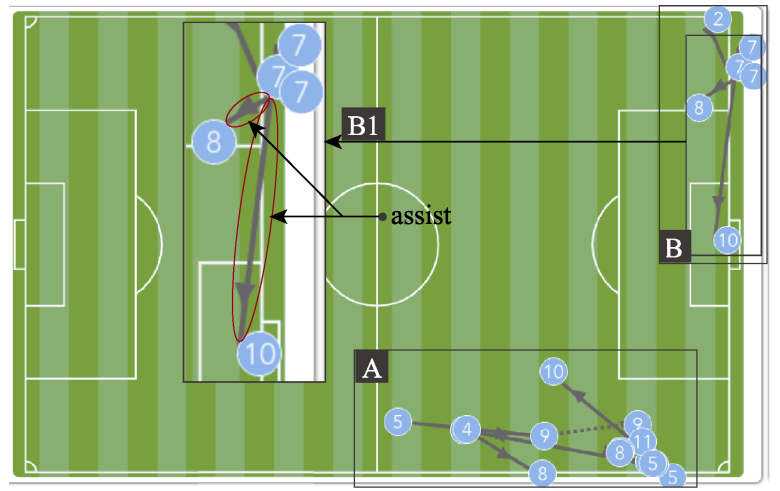

图 4 传球网络对应的空间位置

专家们进一步查看下半场的传球, 以更好地 理解阿根廷如何通过传球策略的转换获得优势. 首先注意到的现象是，上半场频繁出现的球场右 上区域的传球并没有出现在下半场(图 3 中 $\mathrm{B}$ 部 分), 反而出现了多次在球场左侧区域的传球，这 说明了右侧转变到左侧的传球策略的改变. 与此 同时还发现，下半场左侧的传球效率比上半场右 侧的传球效率有了明显的提升，在 3/4 的进攻回合 内都出现了射门的记录(图 3 中 B1 B4 部分), 并成 功地打进 2 个球(图 3 中 B1 部分和 B4 部分). 如图 4 中 B 部分所示, 从 2 个进球的传球网络结构可以 看出, 球队在角球区域附近经过一系列的短传后 成功地创造了得分机会, 并且在这 2 次的进攻中, 7 号球员的传球起到了关键作用(R5). 其中， 7 号球 员的传球洞穿了防守，成功地将球传到了禁区内， 助攻队友完成了射门. 结合网络视图中 7 号球员突
出的统计指标(图 3 中 C 部分)和整体传球网络中较 粗的边 (图 3 中 $\mathrm{D}$ 部分), 专家们进一步确认 7 号球 员是球队进攻的核心

在上述分析中，专家们关注的是几个主要的 进攻回合. 为了更全面、准确地查看传球所在的空 间位置信息以及与传球结构的关系，专家们使用了 球场视图中的热力图查看传球时的空间位置分布. 从图 3 中 $\mathrm{E}$ 部分可以看到, 球队的进攻区域主要在 右侧和左侧, 分别对应了上下半场的分析结论. 综 合以上现象, 专家们认为, 阿根廷将进攻方向从右 侧转换到左侧对比赛胜利起到了积极作用.

\section{3 专家反馈}

专家们肯定了演化视图对分析的积极作用. 图标位置的移动可以帮助他们快速发现传球动态 改变的规律, 帮助其从更细致的角度对足球比赛 进行分析, 从而以更加简便的方式发现影响比赛 结果的关键时间节点和战术变化, 对赛前的准备 和赛后的复盘都起到了提升效果. 其中, 传球图标 的设计也得到了赞赏, 它可以帮助人们同时了解 传球的空间区域、传球网络的统计特征和前后传球 的相似性. 对比常用的视频分析法, 这一工作可以 解放专家们在视频切换上的繁重劳动, 以及兼顾 前后视频的工作记忆负担. 除此之外, 专家们对系 统的进一步提升提出了如下建议. (1) 提高演化视 图中传球网络投影的准确性和可解释性. 投影结 果会对分析的正确性产生极大的影响. 然而, 目前 缺乏一个评估机制来证明投影的正确性，希望未 来可以在系统中展示投影的评估结果. (2) 提供比 赛双方传球的协同分析. 考虑比赛是个博弯的过 程, 希望系统可以无缝地切换到不同球队的传球 进行分析, 并同时对比双方的传球模式, 这样更有 助于挖掘比赛中球队传球战术的关键调整.

\section{4 局限性与未来工作展望}

本文工作的局限性包含以下 2 点.

(1) 未能展现传球过程中空间区域的转换. 目 前球场被切分为 9 块区域. 本文采取了出现最频繁 的空间区域作为某次进攻回合的表示, 然而, 某些 在区域边界之间的传球可能无法被正确地展示. 因此, 未来将设计更合适的空间区域表达, 以展示 传球过程中空间区域的连续变化.

（2）缺乏详细的防守信息. 虽然本文考虑了球 员面临的防守压力对传球的影响, 但对方采取的 防守战术在系统中未得到体现. 未来可以结合自 动的防守检测算法提取出防守战术, 并在系统中 进行深层次的分析, 以理解球队之间的博弯过程. 


\section{4 结 语}

本文通过与足球专家合作总结得到多个关于 分析动态传球网络的领域需求. 根据领域需求, 设 计了足球动态传球网络可视分析系统. 本文结合 基于图标的方法和投影技术，以可视化足球传球 网络，包括网络拓扑结构、相关空间上下文信息以 及重要的网络特征在时间上的动态演变，从而帮助 用户深人分析足球传球网络动态. 最后, 通过案例 分析和专家访谈评估了系统的可用性. 未来考虑在 更多的比赛数据上进行方法的评估与验证.

\section{参考文献(References):}

[1] Yamamoto Y, Yokoyama K. Common and unique network dynamics in football games[J]. PLoS One, 2011, 6(12): e29638

[2] Xie X, Wang J C, Liang H Y, et al. PassVizor: toward better understanding of the dynamics of soccer passes[J]. IEEE Transactions on Visualization and Computer Graphics, 2021, 27(2): 1322-1331

[3] van den Elzen S, Holten D, Blaas J, et al. Reducing snapshots to points: a visual analytics approach to dynamic network exploration[J]. IEEE Transactions on Visualization and Computer Graphics, 2016, 22(1): 1-10

[4] Clemente F M, Couceiro M S, Martins F M L, et al. Using network metrics to investigate football team players' connections: a pilot study[J]. Motriz: Revista de Educação Física, 2014, 20(3): 262-271

[5] Clemente F M, Martins F M L, Wong P D, et al. Midfielder as the prominent participant in the building attack: a network analysis of national teams in FIFA world cup 2014[J]. International Journal of Performance Analysis in Sport, 2015, 15(2): 704-722

[6] Peña J L, Touchette H. A network theory analysis of football strategies[OL]. [2021-07-08]. https://arxiv.org/abs/1206.6904

[7] Bekkers J, Dabadghao S. Flow motifs in soccer: what can passing behavior tell us?[J]. Journal of Sports Analytics, 2019, 5(4): 299-311

[8] Gyarmati L, Kwak H, Rodriguez P. Searching for a unique style in soccer[OL]. [2021-07-08]. https://arxiv.org/abs/1409.0308

[9] Peña J L, Navarro R S. Who can replace Xavi? A passing motif analysis of football players[OL]. [2021-07-08]. http://de.arxiv. org/pdf/1506.07768

[10] Clemente F M, Couceiro M S, Martins F M L, et al. Using network metrics in soccer: a macro-analysis[J]. Journal of Human Kinetics, 2015, 45(1): 123-134

[11] Cotta C, Mora A M, Merelo J J, et al. A network analysis of the
2010 FIFA World Cup champion team play[J]. Journal of Systems Science and Complexity, 2013, 26(1): 21- 42

[12] Duarte R, Araújo D, Folgado H, et al. Capturing complex, non-linear team behaviours during competitive football performance $[J]$. Journal of Systems Science and Complexity, 2013, 26(1): 62-72

[13] Gonçalves B, Coutinho D, Santos S, et al. Exploring team passing networks and player movement dynamics in youth association football[J]. PLoS One, 2017, 12(1): e0171156

[14] Beck F, Burch M, Diehl S, et al. A taxonomy and survey of dynamic graph visualization[J]. Computer Graphics Forum, 2017, 36(1): 133-159

[15] Bach B, Pietriga E, Fekete J D. GraphDiaries: animated transitions and temporal navigation for dynamic networks[J]. IEEE Transactions on Visualization and Computer Graphics, 2014, 20(5): 740-754

[16] Friedrich C, Eades P. The marey graph animation tool demo[C] //Proceedings of the 8th International Symposium on Graph Drawing. Heidelberg: Springer, 2000: 396-406

[17] Forbes A G, Burks A, Lee K, et al. Dynamic influence networks for rule-based models[J]. IEEE Transactions on Visualization and Computer Graphics, 2018, 24(1): 184-194

[18] Archambault D W, Purchase H C, Pinaud B. Animation, small multiples, and the effect of mental map preservation in dynamic graphs[J]. IEEE Transactions on Visualization and Computer Graphics, 2011, 17(4): 539-552

[19] Wu Y H, Pitipornvivat N, Zhao J, et al. egoSlider: visual analysis of egocentric network evolution[J]. IEEE Transactions on Visualization and Computer Graphics, 2016, 22(1): 260-269

[20] Zhao J, Glueck M, Chevalier F, et al. Egocentric analysis of dynamic networks with egolines[C] //Proceedings of the CHI Conference on Human Factors in Computing Systems. New York: ACM Press, 2016: 5003-5014

[21] Burch M, Vehlow C, Beck F, et al. Parallel edge splatting for scalable dynamic graph visualization[J]. IEEE Transactions on Visualization and Computer Graphics, 2011, 17(12): 2344-2353

[22] van den Elzen S, Holten D, Blaas J, et al. Dynamic network visualization with extended massive sequence views[J]. IEEE Transactions on Visualization and Computer Graphics, 2014, 20(8): 1087-1099

[23] Greilich M, Burch M, Diehl S. Visualizing the evolution of compound digraphs with TimeArcTrees[J]. Computer Graphics Forum, 2009, 28(3): 975-982

[24] Wu Y C, Xie X, Wang J C, et al. ForVizor: visualizing spatio-temporal team formations in soccer[J]. IEEE Transactions on Visualization and Computer Graphics, 2019, 25(1): 65-75

[25] Power P, Ruiz H, Wei X Y, et al. Not all passes are created equal: bjectively measuring the risk and reward of passes in soccer from tracking data[C] //Proceedings of the 23rd ACM SIGKDD International Conference on Knowledge Discovery and Data Mining. New York: ACM Press, 2017: 1605-1613 